\title{
A New Lower Bound on the Ergodic Capacity of Distributed MIMO Systems
}

\author{
Michail Matthaiou, Member, IEEE, Nestor D. Chatzidiamantis, Student Member, IEEE, and \\ George K. Karagiannidis, Senior Member, IEEE
}

\begin{abstract}
We present a novel and analytical lower bound on the ergodic capacity of distributed multiple-input multiple-output (D-MIMO) systems operating in composite Rayleigh/lognormal (RLN) fading and assuming double-sided spatial correlation. The proposed lower bound is applicable for finite number of antennas and remains tight across the entire Signal-to-Noise (SNR) regime. In addition, we perform a detailed low-SNR analysis that provides useful insights into the implications of the system parameters on MIMO capacity.
\end{abstract}

Index Terms-Capacity, MIMO systems, shadowing distribution, spatial fading correlation.

\section{INTRODUCTION}

$\mathbf{T}$ HE capacity of point-to-point multiple-input multiple-output (MIMO) communication systems has been extensively explored over the past years under different fading conditions and assuming all different types of spatial correlation [1]-[6]. Recently, there has been growing interest in the area of distributed MIMO (D-MIMO) systems, which combine MIMO technology with the classical principle of distributed antenna systems to make use of both micro and macro-diversity [7]-[10]. In essence, multiple antennas at one end of the wireless channel are packed into multiple radio ports (or nodes) that are spatially separated. Hence, each radio link is affected by different degree of path loss, due to the different access distances, along with different shadowing effects. We point out that the latter manifestation is rather critical when assessing MIMO performance since it can significantly diminish the benefits of MIMO technology.

Surprisingly, little is still known for the capacity performance of D-MIMO systems and this can be attributed to the difficulty in averaging the channel eigen-statistics over the shadowing distribution. In [9], an asymptotic, in terms of antenna elements, capacity analysis was performed that took into account the effects of both spatial correlation and shadowing, while closed-form expressions for the mean/outage spectral efficiency

Manuscript received December 23, 2010; accepted January 26, 2011. Date of publication February 04, 2011; date of current version February 17, 2011. The work of M. Matthaiou was supported in part by the Swedish Governmental Agency for Innovation Systems (VINNOVA) within the VINN Excellence Center Chase. The associate editor coordinating the review of this manuscript and approving it for publication was Prof. Tan Wong.

M. Matthaiou is with the Department of Signals and Systems, Chalmers University of Technology, SE-412 96 Gothenburg, Sweden (e-mail: michail.matthaiou@chalmers.se).

N. D. Chatzidiamantis and G. K. Karagiannidis are with the Department of Electrical and Computer Engineering, Aristotle University of Thessaloniki, 54 124, Thessaloniki, Greece (e-mail: nestoras@auth.gr; geokarag@auth.gr).

Digital Object Identifier 10.1109/LSP.2011.2110645 in the high signal-to-noise (SNR) regime were presented in [10]. The generic approach of [11] used tools from majorization theory to derive upper and lower capacity bounds for Nakagami- $m$ /lognormal D-MIMO systems. Note, however, that the proposed lower bounds in [11, Th. 5, Cor. 6] were given in integral form which the authors approximated numerically via Gauss-Hermite polynomials. More importantly, they are inherently loose in the high-SNR regime and not amenable to further approximations. The lower bound proposed by the authors in [12, Th. 6], though in closed-form, applies for D-MIMO under Nakagami- $m$ /gamma fading which is essentially an analytically friendlier approximation of the Nakagami- $m / \operatorname{lognormal}$ model. This makes clear that a closed-form lower bound on the capacity of Rayleigh/lognormal (RLN) MIMO channels is not yet available. What is more, the effects of spatial correlation were not considered in both [11], [12].

In this letter, we complement and extend the results of [11], [12] to provide a novel and analytical lower bound on the capacity of D-MIMO systems operating in RLN fading. In particular, we consider the joint effects of spatial correlation on both sides of the MIMO link and of large-scale lognormal fading. The proposed general bound is applicable for equal number of antennas at bond ends and remains sufficiently tight across the entire SNR range. For the specific, but still practical, case of semi-correlated Rayleigh fading, the above mentioned symmetry condition is relaxed. Finally, a thorough second-order low-SNR analysis is presented and closed-form expressions are derived for the key parameters determining MIMO performance in this asymptotic regime, namely the minimum energy per bit and wideband slope.

Notation: We use upper and lower case boldface to denote matrices and vectors, respectively. The expectation of a random variable (RV) is given by $\mathcal{E}[\cdot]$. The symbol $(\cdot)^{\dagger}$ represents the Hermitian transpose while the matrix determinant will be interchangeably denoted by $\operatorname{det}(\cdot)$ and $|\cdot|$. Finally, $\operatorname{tr}(\cdot)$ yields the trace of a matrix.

\section{MIMO SYSTEM MODEL}

We consider an uplink D-MIMO system with $N_{r}$ receive antennas at the base station and $L$ radio ports (nodes) each connected to $N_{t}$ transmit antennas and also define $s \triangleq \min \left(L N_{t}, N_{r}\right), t \triangleq \max \left(L N_{t}, N_{r}\right)$. Then, the input-output relationship reads as

$$
\mathbf{y}=\sqrt{\gamma} \mathbf{H} \Xi^{1 / 2} \mathbf{x}+\mathbf{n}
$$

where $\mathbf{x} \in \mathbb{C}^{L N_{t} \times 1}$ and $\mathbf{y} \in \mathbb{C}^{N_{r} \times 1}$ are the transmitted and received signal vectors while $\mathbf{n} \sim \mathcal{C N}\left(\mathbf{0}, \mathbf{I}_{N_{r}}\right)$ is the complex 
AWGN and $\gamma$ corresponds to the average SNR. The matrix $\mathbf{H} \in$ $\mathbb{C}^{N_{r} \times L N_{t}}$ represents the small-scale fading which is assumed to follow a double-sided correlated Rayleigh distribution. As such, we have

$$
\mathbf{H}=\mathbf{R}_{\mathrm{R}}^{1 / 2} \mathbf{H}_{w} \mathbf{R}_{\mathrm{T}}^{1 / 2}
$$

where the entries of $\mathbf{H}_{w}$ are i.i.d. $\mathcal{C N}(0,1)$ RVs while $\mathbf{R}_{\mathrm{R}}, \mathbf{R}_{\mathrm{T}}$ denote the Hermitian, positive definite receive and transmit correlation matrices, respectively. We recall that this product model, referred to as the Kronecker model, has been widely used in the MIMO literature to assess the effects of correlation (e.g., [2]-[5] and references therein). Note that transmit correlation occurs only between the antennas of the same radio port since the $L$ ports are, in general, well separated in space.

The entries of the diagonal matrix $\Xi \in \mathbb{R}^{L N_{t} \times L N_{t}}$ represent the large-scale effects, and hence $\boldsymbol{\Xi}=\operatorname{diag}\left\{\mathbf{I}_{N_{t}} \xi_{m} / D_{m}^{v}\right\}_{m=1}^{L}$ where $D_{m}, m=1, \ldots, L$ denotes the distance between the receiver and the $m$-th radio port while $v$ is the path-loss exponent with typical values ranging from 2 to 6 . As was previously mentioned, in our analysis we model the large-scale fading via the lognormal distribution, which is the prevalent model in the characterization of various radar, optical and RF wireless channels [7]-[11]. In this case, the probability density function of the large-scale fading coefficients reads as

$$
p\left(\xi_{m}\right)=\frac{\eta}{\xi_{m} \sqrt{2 \pi \sigma_{m}^{2}}} e^{-\frac{\left(\eta \ln \xi_{m}-\mu_{m}\right)^{2}}{2 \sigma_{m}^{2}}}, \quad \xi_{m} \geq 0
$$

where $\eta=10 / \ln 10$, while $\mu_{m}, \sigma_{m}$ are the mean and standard deviation (both in $\mathrm{dB}$ ) of the variable's natural logarithm, respectively.

\section{NEW LOWER BOUNDS ON THE ERGODIC CAPACITY OF RLN D-MIMO SYSTEMS}

In the following, we assume that the receiver has perfect channel state information (CSI) while the transmitter has neither statistical nor instantaneous CSI. Then, a sensible choice for the transmitter is to equally split the available power amongst all data streams (i.e. uniform power allocation); then, the MIMO ergodic capacity in bits/s/Hz reads as

$$
C_{\text {erg }}=\mathcal{E}\left[\log _{2}\left(\operatorname{det}\left(\mathbf{I}_{L N_{t}}+\frac{\gamma}{L N_{t}} \mathbf{Z}^{\dagger} \mathbf{Z}\right)\right)\right]
$$

where $\mathbf{Z}=\mathbf{H} \mathbf{\Xi}^{1 / 2}$ and the expectation is taken over all realizations of $\mathbf{H}$ and $\boldsymbol{\Xi}$.

Theorem 1: The ergodic capacity of a symmetric $s \times s$ RLN D-MIMO system with transmit correlation $\mathbf{R}_{\mathrm{T}}$ and receive correlation $\mathbf{R}_{\mathrm{R}}$ is lower bounded by

$$
\begin{gathered}
C_{\text {erg }} \geq C_{\mathrm{L}}=s \log _{2}\left(1+\frac{\gamma}{L N_{t}} \exp \left(\frac { 1 } { s } \left(\ln \left|\mathbf{R}_{\mathrm{R}}\right|+\ln \left|\mathbf{R}_{\mathrm{T}}\right|\right.\right.\right. \\
\left.\left.\left.+\sum_{i=0}^{s-1} \psi(s-i)-N_{t} v \sum_{m=1}^{L} \ln \left(D_{m}\right)+\frac{N_{t}}{\eta} \sum_{m=1}^{L} \mu_{m}\right)\right)\right)
\end{gathered}
$$

where $\psi(x)$ is the Euler's digamma function [13, eq. (8.360.1)].

Proof: The proof relies on the application of Minkowski's inequality to (4), as was originally proposed in [3, Th. 1] and thereafter in [5, eq. (41)], [12, Th. 6]. Omitting explicit details, we can directly lower bound (4) according to

$$
C_{\text {erg }} \geq s \mathcal{E}\left[\log _{2}\left(1+\frac{\gamma}{L N_{t}} \exp \left(\frac{1}{s} \ln \left(\operatorname{det}\left(\mathbf{Z}^{\dagger} \mathbf{Z}\right)\right)\right)\right)\right] .
$$

Recalling that $\log _{2}(1+\alpha \exp (x))$ is convex in $x$ for $\alpha>0$, we employ Jensen's inequality to further lower bound (6)

$$
\begin{aligned}
C_{\mathrm{erg}} \geq & s \log _{2}\left(1+\frac{\gamma}{L N_{t}} \exp \left(\frac{1}{s} \mathcal{E}\left[\ln \left(\operatorname{det}\left(\mathbf{Z}^{\dagger} \mathbf{Z}\right)\right)\right]\right)\right) \\
= & s \log _{2}\left(1+\frac{\gamma}{L N_{t}} \times \exp \left(\frac { 1 } { s } \left(\ln \left|\mathbf{R}_{\mathrm{R}}\right|+\ln \left|\mathbf{R}_{\mathrm{T}}\right|\right.\right.\right. \\
& \left.\left.\left.+\mathcal{E}\left[\ln \left(\operatorname{det}\left(\mathbf{H}_{w}^{\dagger} \mathbf{H}_{w}\right)\right)\right]+\mathcal{E}[\ln (\operatorname{det}(\boldsymbol{\Xi}))]\right)\right)\right)
\end{aligned}
$$

where, from (7) to (8), we have successively used the property of square matrices $\operatorname{det}(\mathbf{A B})=\operatorname{det}(\mathbf{B A})$. We note that $\mathbf{H}_{w}$ is Rayleigh distributed and as such $\mathbf{H}_{w}^{\dagger} \mathbf{H}_{w}$ follows a central (zero-mean) Wishart distribution; then, using [6, Eq. (A.8.1)] we can express the third term in (8) as

$$
\mathcal{E}\left[\ln \left(\operatorname{det}\left(\mathbf{H}_{w}^{\dagger} \mathbf{H}_{w}\right)\right)\right]=\sum_{i=0}^{s-1} \psi(s-i) .
$$

The last term in (8) can be evaluated as follows:

$$
\begin{aligned}
\mathcal{E}[\ln (\operatorname{det}(\boldsymbol{\Xi}))] & =N_{t} \sum_{m=1}^{L} \ln \left(D_{m}^{-v}\right)+N_{t} \sum_{m=1}^{L} \mathcal{E}\left[\ln \left(\xi_{m}\right)\right] \\
& =-N_{t} v \sum_{m=1}^{L} \ln \left(D_{m}\right)+\frac{N_{t}}{\eta} \sum_{m=1}^{L} \mu_{m}
\end{aligned}
$$

where we have used the fact that the term $\ln \xi_{m}$ is Gaussian distributed such that $\mathcal{E}\left[\ln \left(\xi_{m}\right)\right]=\mu_{m} / \eta$. Combining (9), (10) with (8) we can easily obtain (5).

Comparing (4) with (5), we can infer that at high-SNRs the proposed bound becomes exact and equal to

$$
\begin{aligned}
C_{\mathrm{L}}^{\infty}= & C_{\text {erg }}^{\infty}=s \log _{2}\left(\frac{\gamma}{L N_{t}}\right)+\log _{2}\left|\mathbf{R}_{\mathrm{R}}\right|+\log _{2}\left|\mathbf{R}_{\mathrm{T}}\right| \\
& +\frac{1}{\ln 2} \sum_{i=0}^{s-1} \psi(s-i)-N_{t} v \sum_{m=1}^{L} \log _{2}\left(D_{m}\right) \\
& +\frac{N_{t}}{\eta \ln 2} \sum_{m=1}^{L} \mu_{m}
\end{aligned}
$$

which is consistent with the results of [3], [5]. More importantly, this observation is in fundamental contrast with the lower bound in [11], whose tightness degrades as the SNR increases since it is based on a first-order expansion of (4). Note that the above high-SNR expression verifies the well-known characteristic of MIMO systems, that is the asymptotically (in terms of SNR) linear capacity increase with the minimum number of antennas [1], [3], [4], [6].

Moreover, (5) demonstrates that increasing $\mu_{m}$ has a beneficial impact on the ergodic capacity since this scenario corresponds to less severe fading conditions, whereas a higher Tx-Rx distance effectively reduces capacity due to the increased path- 
loss attenuation. On the other hand, $C_{\mathrm{L}}$ is independent of the variance of the shadowing distribution, $\sigma_{m}$.

Corollary 1: The ergodic capacity of a $t \times s$ RLN D-MIMO system with transmit correlation $\mathbf{R}_{\mathrm{T}}$ is lower bounded by

$$
\begin{gathered}
C_{\mathrm{L}}=s \log _{2}\left(1+\frac{\gamma}{L N_{t}} \exp \left(\frac { 1 } { s } \left(\ln \left|\mathbf{R}_{\mathrm{T}}\right|+\sum_{i=0}^{s-1} \psi(t-i)\right.\right.\right. \\
\left.\left.\left.-N_{t} v \sum_{m=1}^{L} \ln \left(D_{m}\right)+\frac{N_{t}}{\eta} \sum_{m=1}^{L} \mu_{m}\right)\right)\right) .
\end{gathered}
$$

Proof: The proof follows trivially by setting $\mathbf{R}_{\mathrm{R}}=\mathbf{I}_{t}$ in (5) and simplifying.

We note that for the simplified case of i.i.d. Rayleigh fading with no shadowing (i.e. $\mathbf{R}_{\mathrm{R}}=\mathbf{I}_{t}, \mathbf{R}_{\mathrm{T}}=\boldsymbol{\Xi}=\mathbf{I}_{s}$ ), the proposed bound coincides with [3, eq. (5)], [5, eq. (78)].

\section{LOW-SNR ANALYSIS}

As was originally pointed out in [14], and later adopted in [2], [12], [15] among others, the low-SNR performance of MIMO systems can be more accurately captured via the normalized transmit energy per information bit $E_{b} / N_{0}$ rather than via the per-symbol SNR. This capacity representation reads as

$$
C_{\text {erg }}\left(\frac{E_{b}}{N_{0}}\right) \approx \mathcal{S}_{0} \log _{2}\left(\frac{\frac{E_{b}}{N_{0}}}{\frac{E_{b}}{N_{0} \text { min }}}\right)
$$

where $\left(E_{b} / N_{0}\right)_{\min }$ and $\mathcal{S}_{0}$ are the two key parameters determining the low-SNR behavior, corresponding to the "minimum normalized energy per information bit required to convey any positive rate reliably" and the wideband slope, respectively. Following [14], these two figures of merit are defined as

$$
\frac{E_{b}}{N_{0} \text { min }}=\frac{1}{\dot{C}_{\mathrm{erg}}(0)}, \quad \mathcal{S}_{0}=-2 \ln 2 \frac{\left(\dot{C}_{\mathrm{erg}}(0)\right)^{2}}{\ddot{C}_{\mathrm{erg}}(0)}
$$

where $\dot{C}_{\text {erg }}(\cdot), \ddot{C}_{\text {erg }}(\cdot)$ denote the first and second-order derivatives of the ergodic capacity (4) over the SNR $\gamma$, respectively. Following [15], the characterization of the low-SNR regime relies on the definition of the dispersion of a random matrix:

Definition 1: For a $M \times M$ random matrix $\mathbf{A}$, its dispersion is defined according to

$$
\zeta(\mathbf{A}) \triangleq M \frac{\mathcal{E}\left[\operatorname{tr}\left(\mathbf{A}^{2}\right)\right]}{\mathcal{E}^{2}[\operatorname{tr}(\mathbf{A})]} .
$$

Theorem 2: For a $N_{r} \times L N_{t}$ RLN D-MIMO system with transmit correlation $\mathbf{R}_{\mathrm{T}}$ and receive correlation $\mathbf{R}_{\mathrm{R}}$, the minimum energy per information bit and the wideband slope are

$$
\begin{aligned}
{\frac{E_{b}}{N_{0} \text { min }}} & =\frac{L \ln 2}{N_{r}}\left(\sum_{m=1}^{L} \frac{1}{D_{m}^{v}} \exp \left(\frac{\mu_{m}}{\eta}+\frac{\sigma_{m}^{2}}{2 \eta^{2}}\right)\right)^{-1} \\
\mathcal{S}_{0} & =\frac{1}{\frac{1}{N_{r}^{2}} \operatorname{tr}\left(\mathbf{R}_{\mathrm{R}}^{2}\right)+\frac{1}{N_{t}^{2}} \mathcal{J}\left(\mathbf{R}_{\mathrm{T}}, \boldsymbol{\Xi}\right)}
\end{aligned}
$$

where

$$
\mathcal{J}\left(\mathbf{R}_{\mathrm{T}}, \boldsymbol{\Xi}\right)=\frac{\sum_{m=1}^{L N_{t}} \frac{\alpha_{m}}{D_{m^{\prime}}^{2 v}} \exp \left(\frac{2 \mu_{m^{\prime}}}{\eta}+\frac{2 \sigma_{m^{\prime}}^{2}}{\eta^{2}}\right)}{\left(\sum_{m=1}^{L} \frac{1}{D_{m}^{v}} \exp \left(\frac{\mu_{m}}{\eta}+\frac{\sigma_{m}^{2}}{2 \eta^{2}}\right)\right)^{2}}
$$

where $m^{\prime}=\left\lceil m / N_{t}\right\rceil$, with $\lceil\cdot\rceil$ being the ceiling operation to the nearest integer, while $\alpha_{m}$ is the $m$-th diagonal entry of $\mathbf{R}_{\mathrm{T}}^{2}$.

Proof: The proof starts by recalling that

$$
\left.\frac{d}{d x} \ln (\operatorname{det}(\mathbf{I}+x \mathbf{A}))\right|_{x=0}=\operatorname{tr}(\mathbf{A})
$$

which implies that we have to evaluate $\mathcal{E}\left[\operatorname{tr}\left(\mathbf{Z}^{\dagger} \mathbf{Z}\right)\right]$. However, a direct evaluation of this term seems to be tedious. In order to circumvent this, we resort to the use of random matrix theory following the reasoning of [15]. In particular, by jointly addressing the effects of transmit side correlation and shadowing we can set $\boldsymbol{\Theta}_{\mathrm{T}}^{1 / 2}=\mathbf{R}_{\mathrm{T}}^{1 / 2} \boldsymbol{\Xi}^{1 / 2}$ in [15, App. B]. Then, the conditions for [15, Lemma 3] to hold are fulfilled, and the desired term simplifies as follows

$$
\begin{aligned}
\mathcal{E}\left[\operatorname{tr}\left(\mathbf{Z}^{\dagger} \mathbf{Z}\right)\right] & =\mathcal{E}\left[\operatorname{tr}\left(\boldsymbol{\Theta}_{\mathrm{T}}\right)\right] \mathcal{E}\left[\operatorname{tr}\left(\mathbf{R}_{\mathrm{R}}\right)\right] \\
& =N_{r} \mathcal{E}\left[\operatorname{tr}\left(\mathbf{R}_{\mathrm{T}}^{1 / 2} \mathbf{\Xi R}_{\mathrm{T}}^{1 / 2}\right)\right] \\
& =N_{r} \mathcal{E}[\operatorname{tr}(\boldsymbol{\Xi})] \\
& =N_{r} N_{t} \sum_{m=1}^{L} \frac{1}{D_{m}^{v}} \exp \left(\frac{\mu_{m}}{\eta}+\frac{\sigma_{m}^{2}}{2 \eta^{2}}\right)
\end{aligned}
$$

where, from (19) to (20), we have exploited the fact that $\boldsymbol{\Xi}$ is diagonal and that the diagonal entries of $\mathbf{R}_{\mathrm{T}}$ are unity, while (21) is a result of the following property of a lognormal variate distributed as $\xi_{m} \sim \mathrm{LN}\left(\mu_{m} / \eta, \sigma_{m}^{2} / \eta^{2}\right)[16]$

$$
\mathcal{E}\left[\xi_{m}^{r}\right]=\exp \left(\frac{r \mu_{m}}{\eta}+\frac{r^{2} \sigma_{m}^{2}}{2 \eta^{2}}\right) .
$$

Combining (4) with (21), we can obtain (15). Likewise, we can express the wideband slope through [15, eq. (19)]

$$
\mathcal{S}_{0}=\frac{2 L N_{t} N_{r}}{L N_{t} \zeta\left(\mathbf{R}_{\mathrm{R}}\right)+N_{r} \zeta\left(\boldsymbol{\Theta}_{\mathrm{T}}\right)} .
$$

Applying the definition (14) in (23), averaging over $\boldsymbol{\Xi}$ with the aid of (22), we obtain (16) after some algebraic manipulations and noting that $\mathbf{R}_{\mathrm{T}}$ is a block-diagonal Hermitian matrix.

Interestingly, the minimum energy per bit is independent of spatial correlation and number of transmit antennas which is in line with the results of [14], [15]. In addition, a higher $N_{r}$ tends to reduce $E_{b} / N_{0_{\min }}$, due to the additional power that is captured via the use of multiple receive antennas. We finally notice that under i.i.d. Rayleigh fading, (15)-(16) simplify to

$$
{\frac{E_{b}}{N_{0} \min }}=\frac{\ln 2}{N_{r}}, \quad \mathcal{S}_{0}=\frac{2 N_{t} N_{r}}{N_{t}+N_{r}}
$$

which respectively coincide with [14, eq. (206)], [15, eq. (17)] and [15, eq. (19)] (i.e. for $\boldsymbol{\Theta}_{\mathrm{R}}=\mathbf{I}_{N_{r}}$ and $\boldsymbol{\Theta}_{\mathrm{T}}=\mathbf{I}_{L N_{t}}$ ).

\section{NUMERICAL RESUlTS}

In this section, the theoretical analysis presented in Sections III and IV is validated through a set of Monte-Carlo simulations. For this reason, we first generate 10000 random realizations of the small and large-scale fading matrices $\mathbf{H}$ and $\boldsymbol{\Xi}$ according to (2) and (3), respectively, and thereafter obtain the simulated ergodic capacity via (4). The transmit correlation matrix is constructed as $\mathbf{R}_{\mathrm{T}}=\operatorname{diag}\left\{\mathbf{R}_{\mathrm{T}, m}\right\}_{m=1}^{L}$ where $\mathbf{R}_{\mathrm{T}, m}$ is the correlation matrix between the antennas of the $m$ th port. The entries of the latter are modeled via the 


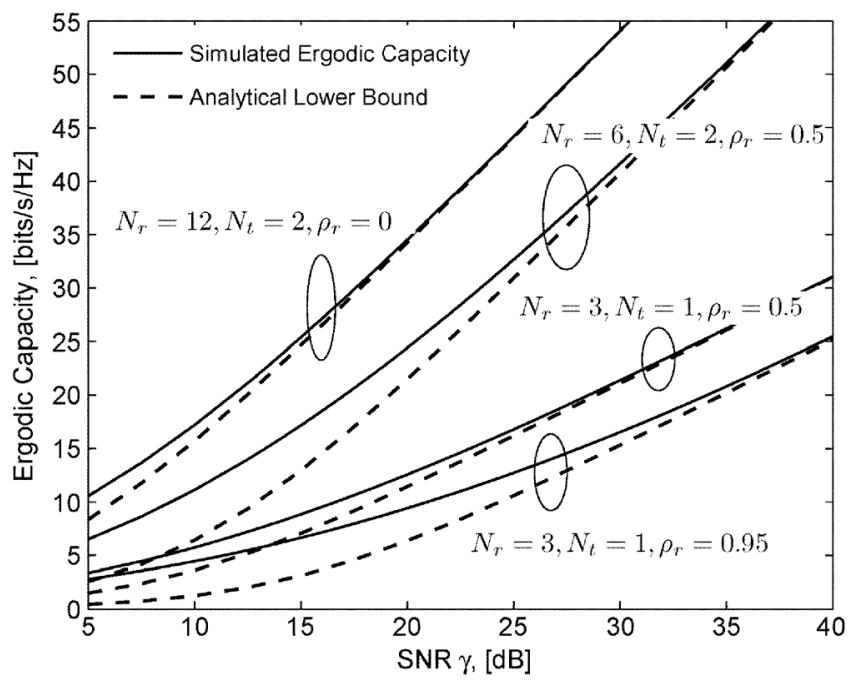

Fig. 1. Simulated ergodic capacity and analytical lower bound against the SNR $\left(L=3, \mu_{m}=2 \mathrm{~dB}, \sigma_{m}=2 \mathrm{~dB}, \rho_{t, m}=0.3, D_{1}=1000 \mathrm{~m}, D_{2}=\right.$ $1500 \mathrm{~m}, D_{3}=2000 \mathrm{~m}$ ).

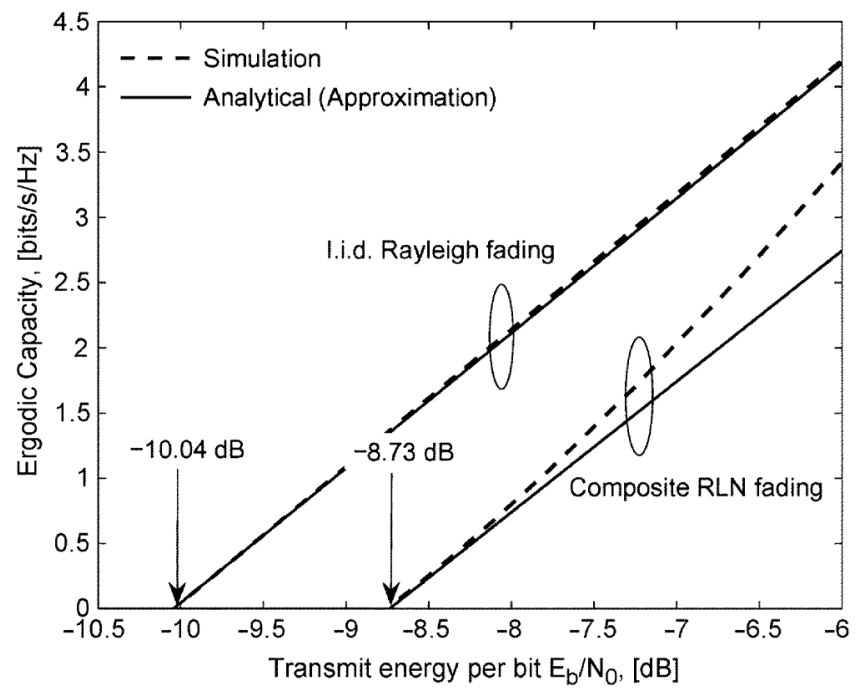

Fig. 2. Low-SNR simulated and analytical ergodic capacity against the transmit energy per bit $\left(N_{r}=7, N_{t}=2, L=3, \rho_{r}=0.5\right)$.

common exponential correlation model $\left\{\mathbf{R}_{\mathrm{T}, m}\right\}_{i, j}=\rho_{t, m}^{|i-j|}$ with $\rho_{t, m} \in[0,1)$ being the transmit correlation coefficient. On a similar basis, we have $\left\{\mathbf{R}_{\mathrm{R}}\right\}_{i, j}=\rho_{r}^{|i-j|}$, where $\rho_{r}$. denotes the receive correlation coefficient. In the following, we set $\mu_{m}=2 \mathrm{~dB}, \sigma_{m}=2 \mathrm{~dB}, \rho_{t, m}=0.3, \forall m=1, \ldots, L$.

In Fig. 1, the simulated ergodic capacity (4) is compared against the analytical lower bounds of Theorem 1, Corollary 1 , for different number of receive/transmit antennas, level of receive correlation and fixed $L=3$. Clearly, spatial correlation diminishes the benefits of MIMO technology due to the reduced spatial diversity. Intuitively, the bound's tightness improves as the difference $\left(N_{r}-L N_{t}\right)$ increases, while for all scenarios under consideration we can notice that the bound becomes exact even at moderate SNR values. As anticipated, at high SNRs, the ergodic capacity increases linearly with the number of antennas whereas in the low-SNR regime, the bound converges asymptotically to the empirical value of ergodic capacity, which is in agreement with [3], [5], [12].
In Fig. 2, the analytical and simulated low-SNR capacity are depicted against the transmit energy per bit $E_{b} / N_{0}$, based on Theorem 2 . For the sake of completeness, the ergodic capacity of a $7 \times 2$ i.i.d. Rayleigh MIMO channel is also overlaid where (24) has been used. We can observe that the presence of lognormal shadowing increases the required minimum energy per bit to a significant extend and slightly decreases the wideband slope. Moreover, the linear approximations seem to remain quite accurate over a moderate range of $E_{b} / N_{0}$ values, especially for the i.i.d. Rayleigh case.

\section{CONCLUSION}

In this letter, we have presented a novel, analytical lower bound on the ergodic capacity of D-MIMO systems in the presence of both double-sided correlated Rayleigh fading as well as large-scale lognormal fading. The derived bound is rather tractable and becomes exact in the low and high-SNR regimes and applies for finite number of antennas. Moreover, a secondorder capacity expansion was investigated at low SNRs and insightful closed-form expressions were derived for the minimum energy per bit and wideband slope.

\section{REFERENCES}

[1] I. E. Telatar, "Capacity of multi-antenna gaussian channels," Eur. Trans. Telecommun., vol. 10, no. 6, pp. 585-595, Nov./Dec. 1999.

[2] S. Jin, M. R. McKay, K.-K. Wong, and X. Li, "Low SNR analysis of multiple-antenna systems with statistical channel state information," IEEE Trans. Veh. Technol., vol. 59, no. 6, pp. 2874-2884, Jul. 2010.

[3] Ö. Oyman, R. U. Nabar, H. Bölcskei, and A. J. Paulraj, "Characterizing the statistical properties of mutual information in MIMO channels," IEEE Trans. Signal Process., vol. 51, no. 11, pp. 2784-2795, Nov. 2003.

[4] H. Shin and J. H. Lee, "Capacity of multi-antenna fading channels: Spatial fading correlation, double scattering, and keyhole," IEEE Trans. Inf. Theory, vol. 49, no. 10, pp. 2636-2647, Oct. 2003.

[5] M. R. McKay and I. B. Collings, "General capacity bounds for spatially correlated Rician MIMO channels," IEEE Trans. Inf. Theory, vol. 51, no. 9, pp. 3121-3145, Sep. 2005.

[6] A. Grant, "Rayleigh fading multi-antenna channels," EURASIP J. Appl. Signal Process., vol. 2002, no. 3, pp. 316-329, Mar. 2002.

[7] W. Roh and A. Paulraj, "Outage performance of the distributed antenna systems in a composite fading channel," in Proc. IEEE Veh. Techn. Conf. (VTC), Vancouver, BC, Canada, Sep. 2002, vol. 3, pp. $1520-1524$.

[8] H. Zhang and H. Dai, "On the capacity of distributed MIMO systems," in Proc. Conf. Inform. Sciences and Systems (CISS), Princeton, NJ, Mar. 2004.

[9] H. Dai, "Distributed versus co-located MIMO systems with correlated fading and shadowing," in Proc. IEEE Int. Conf. Acoustics Speech Signal Proc. (ICASSP), Toulouse, France, May 2006.

[10] D. Wang, X. You, J. Wang, Y. Wang, and X. Hou, "Spectral efficiency of distributed MIMO cellular systems in a composite fading channel," in Proc. IEEE Intern. Conf. Commun. (ICC), Beijing, China, May 2008, pp. 1259-1264.

[11] C. Zhong, K.-K. Wong, and S. Jin, "Capacity bounds for MIMO Nakagami- $m$ fading channels," IEEE Trans. Signal Process., vol. 57, no. 9, pp. 3613-3623, Sep. 2009

[12] M. Matthaiou, N. D. Chatzidiamantis, G. K. Karagiannidis, and J. A. Nossek, "On the capacity of generalized- $K$ fading MIMO channels," IEEE Trans. Signal Process., vol. 58, no. 11, pp. 5939-5944, Nov. 2010.

[13] I. S. Gradshteyn and I. M. Ryzhik, Table of Integrals, Series, and Products, 7th, Ed. San Diego, CA: Academic, 2007.

[14] S. Verdú, "Spectral efficiency in the wideband regime," IEEE Trans. Inf. Theory, vol. 48, no. 6, pp. 1319-1343, Jun. 2001.

[15] A. Lozano, A. M. Tulino, and S. Verdú, "Multiple-antenna capacity in the low-power regime," IEEE Trans. Inf. Theory, vol. 49, no. 10, pp. 2527-2544, Oct. 2003.

[16] J. Aitchison and J. A. C. Brown, The Lognormal Distribution. Cambridge, U.K.: Cambridge Univ. Press, 1957. 